\title{
Message from the Guest Editors of the Special Issue on Solar-Photon Space Sailing
}

\author{
Giovanni Vulpetti ${ }^{1}$, Christian Circi $^{2}$, and Xiangyuan Zeng ${ }^{3}$ \\ 1. International Academy of Astronautics, Paris 75116, France \\ 2. Sapienza University of Rome, Rome 00138, Italy \\ 3. School of Automation, Beijing Institute of Technology, Beijing 100081, China \\ (C) Tsinghua University Press 2019 \\ Dear authors and readers,
}

Since the 1960s, an increasing interest in space propulsion methods $(a)$ not-high-limited in energy (e.g., electric rocket propulsion) and (b) involving no propellant (space sailing), has been advancing from theoretical, numericalsimulation, and technological viewpoints. In particular, among the propulsion concepts of type (b), solar-photon sailing (SPS) has received greater and greater attention.

On May 21st, 2010, at 6:58 (Japan Standard Time), the launcher H-IIA-17 lifted off from the Tanegashima Space Center by carrying two spacecraft as payload. One of those ones is IKAROS, which stands for Interplanetary Kite-craft Accelerated by Radiation Of the Sun. The IKAROS mission is a milestone in the history of astronautics, in general, and of in-space propulsion, in particular. Since then, the efforts of planning and carrying out important almost-propellantless missions have been increasing in objectives and technological improvements, mainly in Japan, USA, China, and Italy.

This special issue hosts many papers by some designers of IKAROS, and Japanese and Italian researchers. Considered the high and spread interest in solar-photon sailing in China, such a collection of important technical papers finds a natural place in the high-quality journal, such as Astrodynamics, which aims at the many astrodynamical areas of modern research.

Part of the papers is devoted to some key sailcraft subsystem technology, and another part regards dynamics, attitude and orbit control, and a task-new micro sail concept. This wide research is not only necessary, but reflects the wide range of problems to be faced with in SPS for achieving a high level of maturity, especially for the realization of the ambitious future space missions.

A final note: the realized IKAROS (JAXA) and NanoSail-D2 (NASA), and the other missions under development in USA and Japan do not yet encompass "standard" solutions to the many key-problems of SPS; merely, it is too early, and at the same time, it is high intriguing just because there is plenty of room for new system/subsystem concepts and related technological levels, and new ideas for extended research/exploration missions.

Sincerely,

Giovanni Vulpetti

Christian Circi

Xiangyuan Zeng

$\bowtie$ G. Vulpetti, g.vulpetti.iaa@gmail.com; C. Circi, christian.circi@uniromal.it; X. Zeng, zeng@bit.edu.cn 